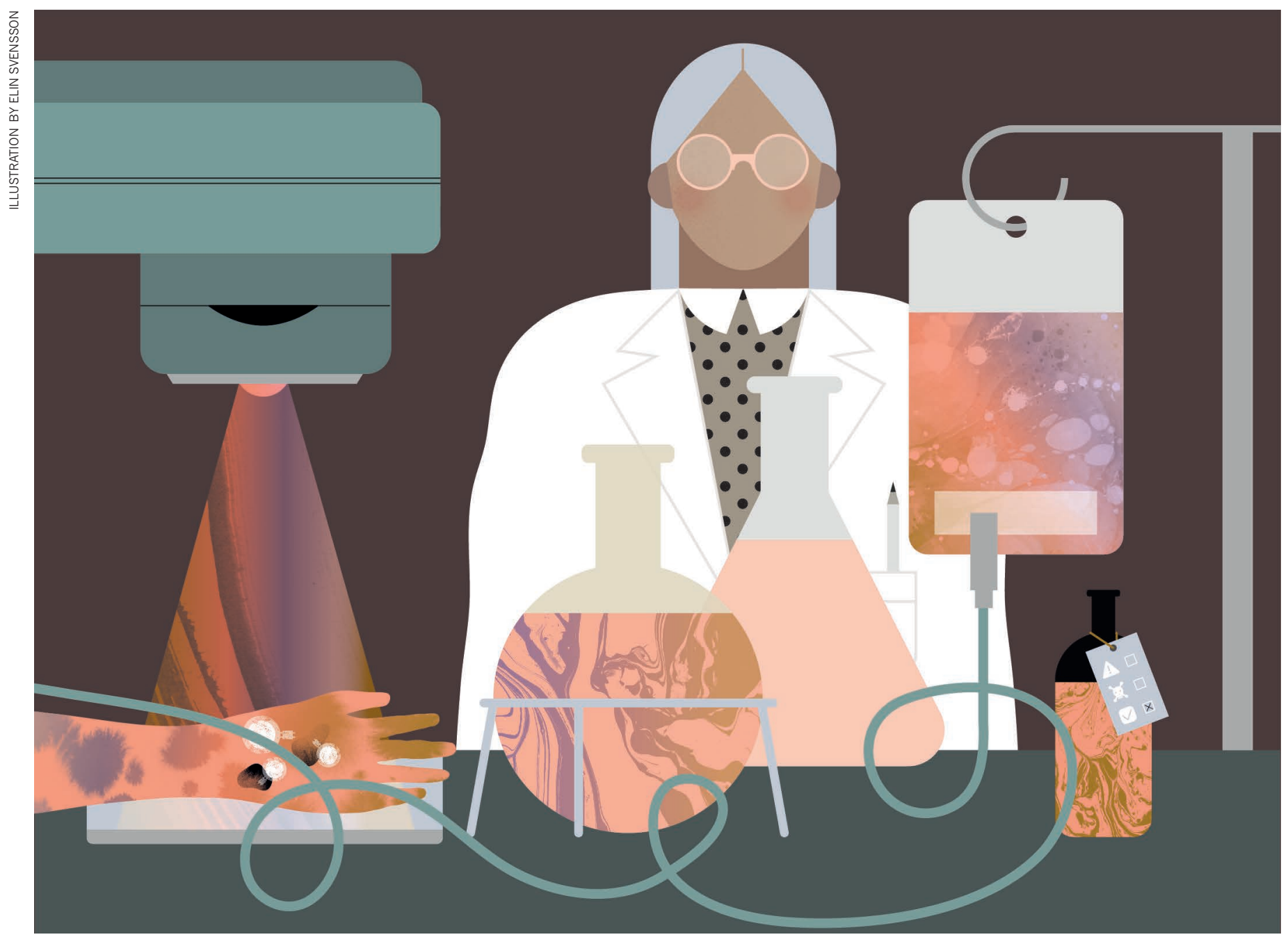

\title{
Combinations on trial
}

\section{By giving immunotherapies in tandem, researchers hope to broaden the benefits.}

\section{BY CHARLES SCHMIDT}

$\mathrm{W}$ hen a patient of Adil Daud started to take an experimental combination of immunotherapy drugs for melanoma in 2015, he had a grapefruit-sized growth bulging under his armpit. The tumour was inoperable, and Daud suspected that the cancer had spread to the 69-year-old man's lungs.

But the two immunotherapeutic agents - the antibodies nivolumab and ipilimumab - had a remarkable effect. "The tumour just melted away," says Daud, an oncologist at the University of California, San Francisco Medical Center. "Within a few weeks, it had vanished."

By giving immunotherapies to people with cancer, doctors hope to spark a self-sustaining attack against cancer cells by immune cells known as $\mathrm{T}$ cells that produces long-term clinical benefits, or even a cure. Around 2,000 immunotherapeutic agents are in development, reports the Cancer Research Institute in New York City. But some people will respond better to the drugs than do others, and many don't respond to them at all. Moreover, tumours can become resistant to such agents over time.

These limitations have pushed immunotherapy researchers towards the use of several drugs in combination. "There's lots of interest in these trials, and people are tripping over each other trying to test as many combinations as possible," says Leena Ghandi, a thoracic medical oncologist at the Laura and Isaac Perlmutter Cancer Center of the New York University Langone Medical Center. Other data gathered by the Cancer Research Institute show that 403 such trials opened worldwide in the first six months of 2017 (see 'In combination: more trials, better targeted'). More than 1,100 are in progress.

By combining immunotherapies, or pairing them with other types of cancer treatment such as chemotherapy or radiation, researchers hope to enhance and broaden the benefits. But combination treatments for cancer that include immunotherapies face specific challenges, both clinical and economic. Immunotherapies send cancer-cell-killing T cells into overdrive, and some trigger dangerous autoimmune reactions that combination treatments can exacerbate. And the cost of the drugs is much greater than that of conventional treatments for cancer - a prescription for a single immunotherapy drug can easily exceed US $\$ 100,000$ per year, so combining them only drives up the expense.

"It's imperative that two- and three-drug regimens lead to clinical benefits that justify 
the added expenses and side effects," says oncologist Jeffrey Weber at the Perlmutter Cancer Center.

\section{CHECKPOINT TAKES THE LEAD}

Immunotherapy drugs take many forms, including antibodies, vaccines, viruses and other agents that make cancer cells more vulnerable to destruction by $\mathrm{T}$ cells. Leading the pack - in terms of both regulatory approval and clinical development - are the treatments known as checkpoint inhibitors.

Immune checkpoints are molecular pathways that evolved to prevent $\mathrm{T}$ cells from turning against healthy tissue - but that tumours can also exploit. Nivolumab targets a checkpoint protein on the surface of T cells called programmed cell death protein 1 (PD-1). When PD-1 binds to its companion protein PD-L1, which is found on the surface of some cancer cells, the $\mathrm{T}$ cell becomes inactivated and the cancer cell avoids attack. By binding to $\mathrm{PD}-1$, nivolumab prevents it binding to PD-L1, and therefore exposes the cancer cell to an immune assault. Other approved drugs achieve the same effect by targeting PD-L1 on the cancer cell. Patrick Ott, an oncologist at the Dana-Farber Cancer Institute in Boston, Massachusetts, estimates that $80-90 \%$ of combination immunotherapy trials involve at least one drug that targets the PD-1 pathway.

Another type of checkpoint inhibitor, of which ipilimumab is an example, targets a checkpoint surface protein called cytoxic T-lymphocyte protein 4 (CTLA-4). But compared with drugs that target PD-1, CTLA-4

inhibitors show greater toxicity. That's because the activity of CTLA- 4 is distributed more widely throughout the body, meaning that its inhibitors may have more harmful autoimmune side effects.

The response of recipients to checkpoint inhibitors also varies by tumour type. According to Ott, $60 \%$ of people with melanoma, $20 \%$ of people with Hodgkin's lymphoma, and 50\% of those with Merkel cell carcinoma (an aggressive form of skin cancer) never respond to treatment that consists of drugs that target only PD-1 or PD-L1. Pairing such drugs with other treatments, Ott says, offers strategies for overcoming the resistance to

\section{"With the right combinations, we might be able to give everyone with cancer abetter chance."} inhibitors of PD-1 or PD-L1 that many recipients show. The tumours of such people might favour other mechanisms of T-cell suppression as well as the PD-1 pathway. Combination treatments therefore offer ways of targeting several such mechanisms simultaneously.

So far, nivolumab with ipilimumab, which targets PD-1 and CTLA-4, is the only checkpoint-inhibitor combination approved for clinical use. It was approved to treat metastatic melanoma by the US Food and Drug Administration (FDA) in 2015, and by the European Medicines Agency the following year.

In 2015, the combination was shown to delay tumour progression in people with melanoma by a median of 11.5 months, almost twice as

\section{IN COMBINATION: MORE TRIALS, BETTER TARGETED}

The number of new clinical trials that combine checkpoint inhibitors targeting PD-1 or PD-L1 with other treatments is soaring worldwide. At the same time, the average planned enrolment for each trial is dropping, partly reflecting more targeted study populations.

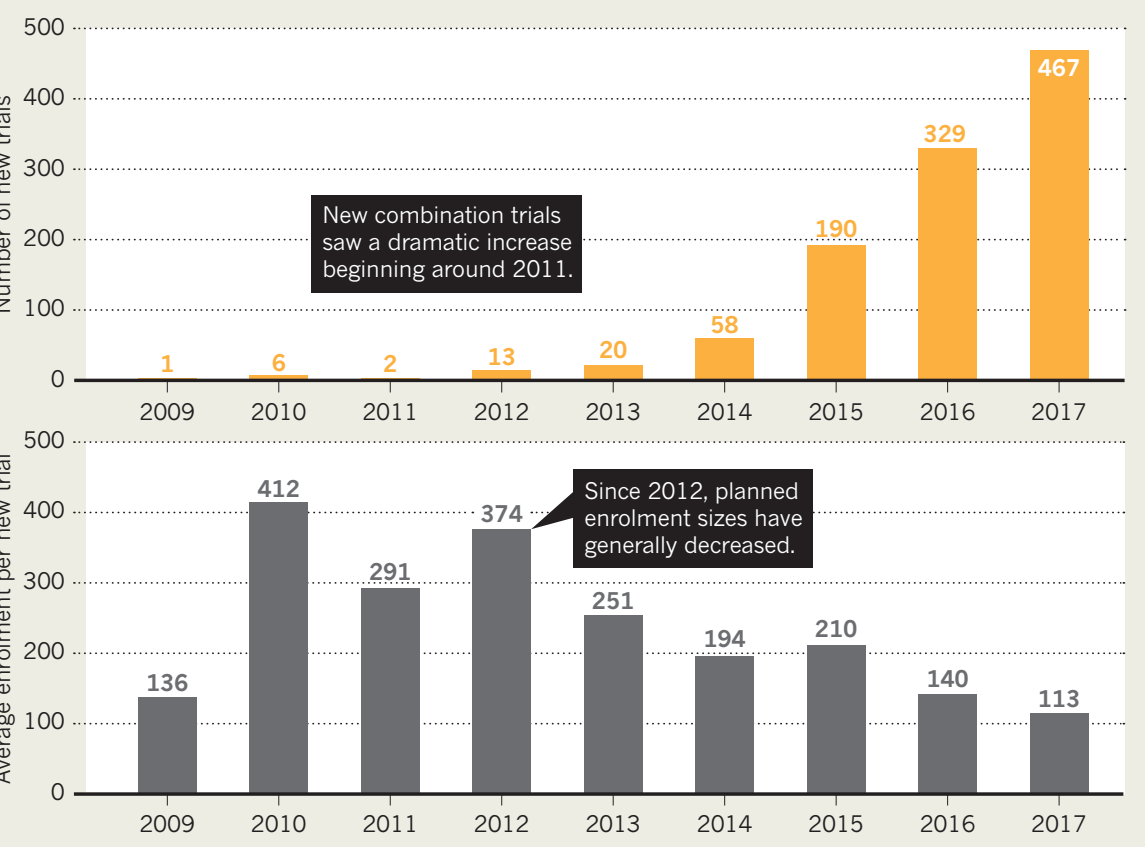

long as in those on nivolumab alone, and almost four times as long as in people treated with only ipilimumab ${ }^{1}$. Then in October 2017, researchers demonstrated that the combination extended survival times: people with melanoma lived longer on the combined treatments, with 58\% still alive after three years compared with $52 \%$ of those treated with nivolumab alone $e^{2}$.

However, the improved rates of survival came with a high burden of toxicity: almost $60 \%$ of people taking the drug combination experienced severe side effects such as colitis or diarrhoea - three times as many as those treated with nivolumab and twice as many as those treated with ipilimumab.

Daud says that researchers were drawn initially to the combination of PD- 1 and CTLA-4 inhibition because drugs with these targets are well established. Ipilimumab was the first checkpoint immunotherapy to reach market (in 2011) and the first two PD-1 inhibitors, pembrolizumab and nivolumab, won regulatory approval in 2014. Combining these two mechanisms just seemed likely to make sense, says Daud.

The combination of PD- 1 and CTLA- 4 inhibitors has since moved into clinical trials for other malignancies, including stomach, breast, bladder, pancreatic, renal, lung and ovarian cancers (see 'Targeted cancer types'). The pairing still makes up the lion's share of immunotherapy combination trials. As of September 2017, 251 such trials were under way, according to the Cancer Research Institute. That tally includes two-way trials combining inhibitors of the PD-1 pathway and CTLA-4, as well as three-way trials combining both of those checkpoint inhibitors with other treatments such as chemotherapy, radiotherapy and targeted drugs that attack cancer cells carrying specific gene mutations.

Despite the large amount of research devoted to this treatment combination and its early promise, researchers are beginning to have serious doubts about its potential. Daud and others cite the toxicity associated with CTLA-4 inhibition as a considerable problem. They also point out that the combination of nivolumab and ipilimumab generally shows only minimal improvement in survival compared with nivolumab alone.

Researchers are paying close attention to trials in people with lung cancer that use an inhibitor of PD-1 or PD-L1 together with a CTLA-4 inhibitor. MYSTIC, a phase III trial conducted by biopharmaceutical company AstraZeneca, based in Cambridge, UK, is testing a combination of the PD-L1 inhibitor durvalumab and a CTLA-4 inhibitor called tremelimumab in people with newly diagnosed lung cancer. But the preliminary results, reported in July 2017, were not overly encouraging - the combination was unable to delay tumour progression for longer than chemotherapy did.

"That was a big disappointment," says Naiyer Rizvi, an oncologist at Columbia University Medical Center in New York City. Rizvi points 
out that the combination may be shown to have improved the overall survival rate of participants when MYSTIC wraps up next year. But he concedes that the data on tumour progression so far don't bode well for better survival rates down the line. That's also a troubling prospect for Bristol-Myers Squibb in New York City, which is sponsoring a phase III trial of nivolumab and ipilimumab in advanced or recurrent non-small-cell lung cancer that should produce results by January 2018 .

\section{AGAINST REGULATIONS}

Other combination trials are targeting different mechanisms of T-cell suppression that tumours use to fend off the immune system. Such mechanisms include the induction of a subpopulation of T cells known as regulatory $\mathrm{T}\left(\mathrm{T}_{\mathrm{reg}}\right)$ cells by the enzyme indoleamine 2,3-dioxygenase (IDO).

$\mathrm{T}_{\text {reg }}$ cells serve a similar function to the $\mathrm{PD}-1$ and CTLA- 4 checkpoint pathways in protecting the body from autoimmune diseases, but also protect tumours from an immune attack. Tumours filled with $\mathrm{T}_{\text {reg }}$ cells suggest a poor prognosis, and the number of $\mathrm{T}_{\text {reg }}$ cells rises as IDO activity increases.

One inhibitor of IDO that has been shown to suppress $\mathrm{T}_{\text {reg }}$ cells is indoximod, developed by NewLink Genetics of Ames, Iowa. In April 2017, Yousef Zakharia, a medical oncologist at the University of Iowa in Iowa City, reported findings from a phase II trial of indoximod in combination with the PD-1 inhibitor pembrolizumab in people with advanced melanoma ${ }^{3}$. The combined treatments shrank tumours in $52 \%$ of those treated. Zakharia points out that the result was much better than the $34 \%$ response rate reported by a trial in 2015 in which people with advanced melanoma received only pembrolizumab ${ }^{4}$. That trial was sponsored by pharmaceutical company Merck in Kenilworth, New Jersey, which makes pembrolizumab.

What's more, Zakharia says, the combination of indoximod and pembrolizumab was better tolerated than that of the FDA-approved nivolumab and ipilimumab. But the results should be interpreted cautiously, Zakharia emphasizes, until they are confirmed by further studies.

\section{TURNING COLD TUMOURS HOT}

Apart from nivolumab-ipilimumab for advanced melanoma, there is only one other FDA-approved combination treatment: the pairing of immunotherapy drug pembrolizumab with chemotherapy in people newly diagnosed with metastatic non-small-cell lung cancer. The results of a Merck-sponsored phase II trial showed that those treated with the combination, which was approved by the FDA in May 2017, survived for a median of 19 months before their cancer got worse, compared with 8.9 months in people treated with chemotherapy alone.

\section{TARGETED CANCER TYPES}

Lung cancer and melanoma, two forms of cancer with a high level of genetic mutation, are the most common target of combination trials of immunotherapy drugs in the past eight years.

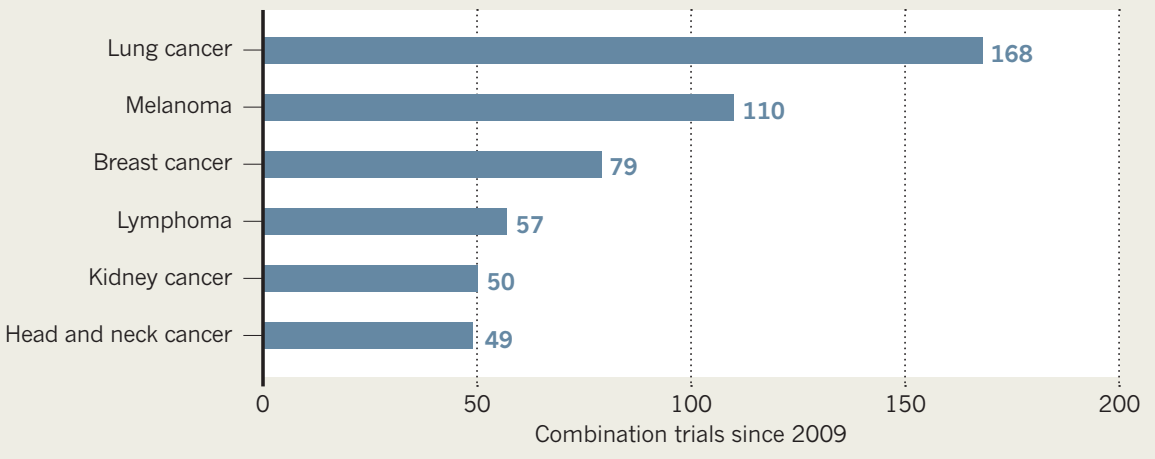

The idea behind the combination is that by killing malignant cells, the chemotherapy generates an inflammatory response that draws $\mathrm{T}$ cells towards the cancer. Pembrolizumab can then disable the PD-1 checkpoint pathway that protects tumours from an immune attack. Ott explains that checkpoint inhibitors work mainly in 'hot' tumours that have already been recognized by the immune system, and they do not induce a response to 'cold' tumours that haven't been identified. The chemotherapy, in effect turns a cold tumour 'hot' so that T cells can move in for the kill, with PD-1 inhibitors helping to clear their path.

Chemotherapy offers just one of several strategies for triggering the immune response on which inhibitors of PD-1 or PD-L1 rely. Radiotherapy, for instance, induces tumour inflammation and boosts the activity of checkpoint inhibitors, as do vaccines made from proteins or other substances (antigens) produced by tumours that can then be injected back into the body. "Even the cells that aren't destroyed by these therapies upregulate stress responses that change how the tumour looks to the immune system," says James Gulley, a medical oncologist at the US National Cancer Institute in Bethesda, Maryland. "It's as if they shine a spotlight on the tumour to facilitate better immune-mediated cancer-cell killing."

Other approaches include vaccines made from neoantigens - mutant proteins derived from the patient's tumour (see page S76). For instance, Ott and his colleagues reported data from a phase I trial that showed that a combination of PD-1 inhibitors and a neoantigenbased vaccine produced long-term remission in people with advanced melanoma ${ }^{5}$.

Another trial in people with melanoma is pairing pembrolizumab with a virus called talimogene laherparepvec (T-VEC) that preferentially infects cancer cells. A genetically engineered form of herpesvirus, T-VEC replicates in infected cells until they break apart and release tumour antigens into the blood that attract $\mathrm{T}$ cells. Researchers on the trial, which is being sponsored by Amgen of Thousand Oaks, California, reported in September that the combination of pembrolizumab and T-VEC shrank melanoma tumours to a greater degree than did either treatment alone ${ }^{6}$.

\section{UNPREDICTABLE BENEFIT}

The effect of immunotherapy varies considerably from person to person. That diversity of response has led to the crucial need to identify better biological markers (biomarkers) for predicting how individuals will react to specific treatments (see page S72) The need is particularly acute in combination immunotherapy, given its higher cost and extra toxicity. The most important biomarker for predicting whether a person will respond to PD-1 inhibitors is the expression of PD-L1. Roy Baynes, senior vice-president of global clinical development at Merck, says that microsatellite instability, a genetic condition caused by impaired DNA repair, can also help to predict who will respond to PD-1 inhibition. "Neither is perfect, but they are helpful," he says.

The hundreds of combination trials that are starting each year will soon deliver an avalanche of data that should yield insight on how best to harness anticancer immunity in each person. An improved understanding of how tumours and the immune system interact should help to make the promise of new therapeutic combinations a reality.

"We're seeing more patients reach five-year survival on immunotherapy, and our hope is that with combination treatments we'll increase that overall number," says Gandhi. "With the right combinations, we might be able to give everyone with cancer a better chance."

Charles Schmidt is a freelance science writer based in Portland, Maine.

1. Larkin, J. et al. N. Engl. J. Med. 373, 23-34 (2015).

2. Wolchok, J. D. et al. N. Engl. J. Med. 377, 13451356 (2017).

3. Zakharia, Y. et al. Proc. AACR Ann. Meet. 77 (Suppl.), CT117 (2017)

4. Robert, C. et al. N. Engl. J. Med. 372, 2521-2532 (2015).

5. Ott, P. A. et al. Nature 547, 217-221 (2017)

6. Long, G. V. et al. J. Clin. Oncol. 34 (Suppl.) 95689568 (2016). 


\section{CORRECTION}

The Outlook article 'Combinations on trial' (Nature 552, S67-S69; 2017) overstated the number of immunotherapeutic agents in development. There are roughly 2,000 , not more than 2,400 . 